\title{
Clinical Curative Effect Observation of Treatment in Vertebral Artery Type of Cervical Spondylopathy with Traditional Chinese Medicine Comprehensive Therapy
}

\author{
Wang Yanan, Tian Jing, Bai Junxiang \\ Shijiazhuang Medical College, Hebei, Shijiazhuang, 050000
}

Keywords: Vertebral artery type cervical spondylitis; Traditional Chinese medicine comprehensive therapy; Clinical efficacy

\begin{abstract}
In order to investigate the clinical effect of comprehensive therapy of traditional Chinese medicine on cervical spondylitis of vertebral artery type, a total of 120 patients with vertebral artery type cervical spondylitis treated in our hospital from September 2017 to March 2018 were selected as the study subjects. The patients were randomly split into a control group and an experimental group, with 60 patients in each group. Patients in the control group were treated with Western medicine. Patients in the experimental group were given ample treatment methods such as massage, acupuncture, and cupping. The clinical treatment effects and improvement of arterial blood flow were compared between the two groups. The treatment effect of the experimental group was significantly greater than that of the control group, and the arterial blood flow improvement in the experimental group was significantly better than that in the control group. The $p$ value less than 0.05 was statistically significant. The adoption of TCM comprehensive therapy for the treatment of vertebral artery type cervical spondylitis can significantly improve the therapeutic effect, and can significantly improve the arterial blood flow in patients. It is worth promoting in the clinical practice.
\end{abstract}

With the continuous changes in people's lifestyles and the increasing pressure of living and work, the clinical incidence of cervical spondylitis presents a trend of year-by-year growth and youthfulness. It has grown up to be a common and frequently-occurring disease in orthopaedics department. The patient's normal work and life has been seriously affected. Vertebral arterial cervical spondylitis, in fact, is the patient's cervical bone and its soft tissue appears non-specific inflammatory hyperplasia, resulting in compression of the vertebral artery and blood flow phenomenon, resulting in a series of symptoms of the nervous system syndrome, which has a serious impact on the patient's physical health and quality of life [1]. This paper adopts the comprehensive treatment of traditional Chinese medicine for the treatment of vertebral artery type cervical spondylitis, and has achieved good clinical results.

\section{Data and Methods}

120 patients with vertebral artery type cervical spondylitis who were admitted to our hospital from September 2017 to March 2018 were selected as the study subjects. According to the random principle, they were divided into a control group and experimental group, with 60 patients in each group. 120 patients selected in this article have been identified as Vertebral Artery Type of Cervical Spondylosis by the "Guidelines for the Clinical Study of New Chinese Medicines." In the control group, there were 28 male patients and 32 female patients. The age range was 39 to 76 years, and the average age was $(42.7 \pm 2.6)$ years. The disease duration was 2 to 13 years, and the average disease duration was ( $5.7 \pm 1.8$ years); In the experimental group, there were 31 male patients and 29 female patients. The age range was 39 to 74 years, and the average age was (41.3 \pm 2.3$)$ years. The disease duration was 1-14 years, and the the average was (6.1 \pm 1.9 years). There was no significant differences in gender, age, duration of disease between the two groups, and they were comparable $(\mathrm{P}>0.05)$. 
Control group: Patients in the control group was treated with Western medicine. All patients in the control group were treated in western medicine, Xizhitin intravenously infusion of 250ml, once daily; Sipilene 10mg orally, once daily. If the patient's cervical spine is not suitable, the patient should be permitted to wear a neck to stop the activity when the patient gets up or sitting. The braking time is generally about 15 days. Nursing staff will demonstrate the correct working posture of the neck to the patient, and the paralyzed patient will continue to carry out neck exercises twice a day without feeling pain or dizziness.

Experimental group: Patients in the experimental group received comprehensive treatment methods such as massage, acupuncture, and cupping.

The first is massage therapy. One is rubbing and pinching. Nursing staff can use massage to massage the wind pool, Fengfu, Dazhui, and neck 1 to neck 7 of the patient. Each point and site are rubbed for three minutes. Each acupoint and site are knead for 80 times in one minute. At the same time, the nursing staff can take the patient's shoulder well point treatment, the number of massages is 60 times per minute, at the same time, the nursing staff should also make a thumb point on the patient's Baihui, brain empty, brain household three acupuncture points. Each point is pressed for 3 minutes, and the number of presses in per minute is 100 times ${ }^{[2]}$. The second is physical reinforcement. Firstly, the medical staff need to let the patient to relax the trapezius muscle, the occipital muscle, and the sternocleidomastoid muscle and then reset it.

The nursing assistant can assist the patient's shoulders and ask the patient to lift his head to fight the force exerted by the traction. Nursing staff can use the left hand to keep the patient's jaw, and then use the right hand to press the patient's Fengchi acupoint. At the same time, the thumb should be seen in the vertebral transverse process where the vertebral body needs to be reset, and then the longitudinal axis of the cervical lordosis. The applied pull traction force is opposed. The third is the leader and brain. The caregiver sticks paperlessly to the patient's scalp and massages the patient's head from front to support. The speed can be altered slowly. Afterwards, the nursing staff can use the thumb to press the of the patient's Baihui, Temple and Yintangacupuncture points. The time for each acupuncture point was three minutes and the frequency of was 100 beats/minute.

Followed by acupuncture and cupping therapy. The acupuncture and cupping therapy was comprehensively applied on the basis of massage therapy for the experimental group. The first is acupuncture. The medical staff can select the patients's acupuncture points such as Fengchi, Jianjing, Dazhui, and Jiangu for acupuncture. After inserting the needle, the needle can be eliminated and the needle can be kept for half an hour. The second is cupping therapy, cupping with flash tank method, leaving the bank for five minutes, if the patient has skin allergies, ulceration and other symptoms cannot use cupping therapy. Cupping therapy once/day for a period of ten days ${ }^{[3]}$.

Patients in the control group were addressed in Western medicine. Patients in the experimental group were given comprehensive treatment methods such as massage, acupuncture, and cupping, and the dispassionate treatment effects and improvement of arterial blood flow were compared between the two groups. According to the criteria for determining the efficacy of TCM diagnostic criteria, the patients were cured: the patient's cervical spondylitis fully recovered and the patient's neck and shoulder movements were completely restored. Markedly effect: The patient's symptoms and signs of cervical spondylitis were significantly controlled and the patient's neck and shoulder movements were close to normal. Effective: Patients with signs and symptoms of cervical spondylitis and the patient's shoulder and neck movement still have some obstacles. Invalid: The patient's symptoms and signs of cervical spondylitis, and shoulder and neck movements have not improved and symptoms of deterioration have occurred. At the same time, the blood flow in the left vertebral artery and the right vertebral artery and the basilar artery of the patient were observed by using cranial Doppler ultrasound.

All data in this paper will be dealt with and analyzed by SPSS 18.0 software. The metrological data is represented by mean \pm . $\mathrm{P}<0.05$ is considered statistically significant. 


\section{Result}

Table 1. Comparison of overall treatment effects between two groups of patients

\begin{tabular}{llllll}
\hline Group & $\mathrm{n}$ & \multicolumn{4}{l}{ Treatment effect } \\
\cline { 3 - 6 } & & Recovered & Effective & Ineffective & Total effective rate \\
\cline { 4 - 6 } $\begin{array}{l}\text { Contrast } \\
\text { group }\end{array}$ & 6 & 12 & 34 & 14 & $46(76.7 \%)$ \\
\hline $\begin{array}{l}\text { Experimental } \\
\text { group }\end{array}$ & 6 & 23 & 33 & 4 & $56(93.3 \%)$ \\
P Value & 0 & & & & \\
\hline
\end{tabular}

It can be seen from Table 1: In the experimental group, 23 cases of patients were healed, 33 cases of patients were effective, and 4 cases of patients were ineffective. The total effective rate of treatment in the treatment group was 93.3\%. In the contrast group, 12 cases of patients were recovered, 34 cases of patients were effective, and 14 cases of patients were ineffective. The total effective rate of treatment in the contrast group was just $76.7 \%$. Thus, in terms of therapeutic effect, patients in the treatment group were significantly better than those in the contrast group, and $\mathrm{P}<0.05$ was considered statistically significant.

Table 2. Comparison of blood flow between two groups of patients

\begin{tabular}{lllll}
\hline Blood folw & & Contrast group & $\begin{array}{l}\text { Experimental } \\
\text { group }\end{array}$ & P Value \\
\hline Left vertebral & Pre-treatment & $21.1 \pm 6.2$ & $21.3 \pm 5.9$ & $<0.05$ \\
artery & Post treatment & $22.0 \pm 6.1$ & $25.7 \pm 6.5$ & \\
Right vertebral & Pre-treatment & $13.1 \pm 5.2$ & $13.4 \pm 4.9$ & $<0.05$ \\
artery & Post treatment & $14.1 \pm 3.4$ & $17.6 \pm 3.8$ & \\
Arteriae & Pre-treatment & $52.1 \pm 12.3$ & $52.0 \pm 11.9$ & $<0.05$ \\
basilaris & Post treatment & $55.7 \pm 9.3$ & $58.7 \pm 10.2$ & \\
\hline
\end{tabular}

It can be seen from Table 2: Prior to treatment, there was no significant differences in blood flow velocity between the left vertebral artery, the right vertebral artery and the basilar artery in both groups. After treatment, the blood flow velocity in the left vertebral artery, the right vertebral artery, and the basilar artery were improved in both groups. And the improvement in blood flow velocity in the experimental group was significantly better than that in the contrast group. The $\mathrm{p}<0.05$ was considered statistically significant.

\section{Conclusion}

Vertebral artery type cervical spondylitis is also called cervical vertigo. The main clinical symptom is episodic vertigo, which has a profound impact on the patient's normal work and life. At present, the pathogenesis of vertebral artery type cervical spondylitis is not completely clear, but most scholars believe that the pathogenesis of vertebral artery type cervical spondylitis is mainly due to destabilization caused by degeneration of cervical intervertebral disc or cervical spine, and thus to the vertebral artery. The sympathetic nerve produces stimulation vertically, which in turn causes stenosis of the vertebral artery. For the treatment of the disease, general therapy is mainly used for the treatment at the present stage and has achieved excellent clinical results. This study shows that in the treatment effect, in the experimental group, 23 patients were cured, 33 patients were effective, and 4 patients were ineffective. The total effective rate of the treatment group was 93.3\%. In the control group, 12 patients recovered, 34 patients were effective, and 14 patients were invalid. The total effective rate of treatment in the control group was only $76.7 \%$. Thus, in terms of therapeutic effect, patients in the treatment group were significantly better than those in the control group, and $\mathrm{P}<0.05$ was examined statistically significant. It also shows that in the blood flow situation, prior to treatment, there was no significant differences in blood flow velocity between the 
left vertebral artery, the right vertebral artery, and the basilar artery in both groups. After the treatment, the blood flow velocity of the left vertebral artery, the right vertebral artery and the basilar artery were improved in both groups, and the improvement of the blood flow velocity in the experimental group was significantly better than that in the control group. The p value difference was lower than 0.05 and tatistically significant.

In short, the adoption of comprehensive Chinese medicine therapy for the treatment of vertebral artery type cervical spondylitis can significantly improve the therapeutic effect and patient's arterial blood flow, which is worthy of promotion and application in clinical practice.

\section{References}

[1] Qingying Meng. Traditional Chinese medicine comprehensive treatment of 104 cases of vertebral artery type of cervical spondylitis [J]. Hunan Traditional Chinese Medicine, 2014,30 (1) :71-72.

[2] Junpeng Zhang. Therapeutic analysis of inclusive treatment of vertebral artery type cervical spondylopathy with traditional Chinese medicine[J]. Massage and Rehabilitation Medicine, 2015,6(7) :37-38.

[3] Chun Zhang. Acupuncture cupping and massages combined treatment of vertebral artery type cervical spondylitis in 25 cases [J]. Chinese Medicine Modern Distance Education, 2012,10 (20): 48-49. 